\title{
RIQUEZA FLORISTICA DEL SANTUARIO DE LA NATURALEZA PALMAR EL SALTO, COMUNA DE VIÑA DEL MAR, REGIÓN DE VALPARAISO, CHILE
}

\author{
FLORAL RICHNESS OF THE NATURE SANCTUARY PALMAR EL SALTO, VIÑA \\ DEL MAR, VALPARAISO REGION, CHILE
}

\author{
Lorena Flores-Toro \& Francisco Aguirre-Saavedra \\ Instituto de Biología, Pontificia Universidad Católica de Valparaíso, Casilla 4059, Valparaíso, Chile. \\ lflorestoro@vtr.net
}

\begin{abstract}
RESUMEN
El Santuario de la Naturaleza Palmar El Salto alberga la tercera población de Jubaea chilensis (Molina) Baillon (Palma chilena), más numerosa del país. El objetivo de este trabajo es contribuir al conocimiento de la flora presente en el Santuario. Se trabajó con 30 censos de vegetación levantados con la metodología fitosociológica de la escuela de ZurichMontpellier. La flora está compuesta por 234 especies. En la flora domina el elemento endémico con 92 especies. Las especies más importantes en el palmar son: Nassella chilensis (Trin.) E.Desv., Chusquea cumingii Nees, Retanilla trinervia (Gillies et Hook.) Hook. et Arn. yJubaea chilensis, todas autóctonas. Destaca la presencia de nueve especies con problemas de conservación, la mayoría vulnerables a la extinción.
\end{abstract}

Palabras Claves: palmares, flora, endemismo.

\section{ABSTRACT}

The Nature Sanctuary "Palmar El Salto", is home to the third largest Jubaea chilensis (Molina) Baillon (Chilean palm tree) population in the country. The objective of this study is to contribute to the knowledge of the flora found in the sanctuary and, to achieve this, thirty vegetation census were carried out with the phytosociologic method of the Zurich-Montpellier school. The flora consists of 234 species. Conformed by 92 species, the endemic element is dominant in the flora. The most important species in the palm grove are: Nassella chilensis (Trin.) E.Desv., Chusquea cumingii Nees, Retanilla trinervia (Gillies et Hook.) Hook. et Arn. and Jubaea chilensis, all native. Stands out the presence of nine species with conservation problems, most of them vulnerable to extinction.

KEYwords: palm grove, flora, endemism.

\section{INTRODUCCION}

Las comunidades de Jubaea chilensis, se encuentran enmarcadas geográficamente en el tipo forestal esclerófilo, sin embargo sus características fisonómicas tan particulares, determinan su clasificación como tipo forestal Palma Chilena (Donoso 1981). En el pasado, los palmares se distribuían en densas poblaciones desde el Río Limarí (Región de Coquimbo) al Río Maule (Región del Maule), distribución que actualmente se encuentra muy reducida y de la cual sólo quedan poblaciones fragmentadas, con muy pocos individuos (González et al. 2001). Hoy en día, las poblaciones más numerosas de palmas según Gallardo etal. (2006) se encuentran en Ocoa (Parque Nacional La Campana en la Región de Valparaíso), con más de 62.000 ejemplares desde infantiles hasta adultos, seguidas por las poblaciones de Cocalán, en terrenos privados, en la Región del 
Libertador General Bernardo O’Higgins con unos 25.000 individuos. En tercer lugar, se encontraría el Palmar El Salto, en la Región de Valparaíso, con 6.061 ejemplares adultos de acuerdo al censo realizado por Portal \& Calderón (1995), el cual sirvió como antecedente para que el lugar fuera declarado Santuario de la Naturaleza por el Ministerio de Educación el año 1998.

Hasta el momento los estudios ecológicos en el sector Palmar El Salto se han centrado fundamentalmente en describir la estructura de la población de palma y su densidad (Michea 1988; Portal \& Calderón 1995; González et al. 2001). Otros autores, como Quintanilla \& Reyes (1999) dan cuenta de la alteración que ha sufrido la vegetación por efectos del fuego en sectores donde hay palmas. Redón (2003), estudió la flora de la cuenca del estero de Viña del Mar, trabajo que incluye cerros y quebradas vecinos al Palmar El Salto, sin embargo no es un estudio referido específicamente a la flora del santuario.

Llama la atención la ausencia de estudios florísticos en el Palmar El Salto si se considera que en los últimos quince años se han desarrollado una serie de obras civiles de gran envergadura que han alterado drásticamente el paisaje del lugar. Entre los años 1994 y 1997 se construyó la carretera Las Palmas que cruza los cerros y quebradas del sector, en el año 1999 se instaló un gasoducto que pasa por algunas laderas del palmar para llevar gas de cañería a Viña del Mar. Por último, se instalaron torres de alta tensión en las partes más altas de estas cuencas. En todos los casos mencionados, según nuestras observaciones personales, no sólo se removió una gran cantidad de terreno para llevar a cabo las obras, sino que además se habilitó caminos en medio de las laderas para facilitar el acceso de maquinarias y camiones para el transporte de materiales, abriéndose una verdadera red de caminos de penetración, destruyendo irreversiblemente la cubierta vegetal en los sectores intervenidos, alterando la composición florística y dando paso a la erosión.

Debido a que la información sobre las plantas vasculares que habitan en el Santuario de la Naturaleza Palmar El Salto es escasa e incompleta, esta contribución tiene como objetivo aportar al conocimiento científico básico que debe ser considerado antes de realizar cualquier intervención antrópica en el palmar o sus inmediaciones, y ser utilizado además para el monitoreo de la biodiversidad de este ecosistema en el futuro.
Área de ESTUdio

El Santuario de la Naturaleza Palmar El Salto (33 03'3305' S; $71^{\circ} 30^{\prime}-71^{\circ} 32^{\prime}$ W) se localiza en la comuna de Viña del Mar, Región de Valparaíso, Chile (Figura 1). Abarca una superficie de 328 ha fragmentadas en 4 áreas: Rodelillo (Altos de Quinta Vergara) con 82 ha, Altos del Tranque (Forestal) con 26 ha, Quebrada Las Siete Hermanas con 54 ha y Quebrada El Quiteño con 166 ha. (Möder et al. 1997). El sitio de estudio se centra en las dos últimas quebradas por presentar las mayores concentraciones de palmas. Éstas quebradas se orientan de sur a norte, con alturas que oscilan entre los 90 y 250 m.s.n.m., son angostas y con pendientes fuertes. Ambas presentan una red de drenaje, que confluye en un sólo cauce permanente que desemboca en el estero Marga-Marga, aunque con una amplia variación estacional debido al régimen pluviométrico típico del clima mediterráneo, con precipitaciones que alcanzan los $400 \mathrm{~mm} / \mathrm{año}$, concentradas en los meses de invierno. Las temperaturas oscilan entre $6-18^{\circ} \mathrm{C}$ en invierno y $14-27^{\circ} \mathrm{C}$ en verano. Los suelos derivan directamente de la meteorización física y química de las rocas graníticas presentes en el área, lo que sumado a las fuertes pendientes origina un suelo con escasa materia orgánica y muy propenso a la erosión (Portal \& Calderón 1995).

\section{METODOS}

El catálogo florístico se confeccionó a partir de colectas intensivas realizadas durante los meses de octubre a diciembre de 2003, julio a octubre de 2006 y abril de 2007 con objeto de encontrar la mayor cantidad especies en flor para su posterior determinación. Las especies fueron colectadas, herborizadas y posteriormente determinadas, utilizando la literatura pertinente y comparándolas con ejemplares de los herbarios de la Pontificia Universidad Católica de Valparaíso (UCVA) y del Museo Nacional de Historia Natural (SGO). La nomenclatura utilizada sigue a Marticorena \& Quezada (1985), excepto los casos en que se adoptaron combinaciones válidas con posterioridad. Los nombres comunes se tomaron de Baeza (1930) y Gajardo (1994). El origen fitogeográfico se obtuvo de la literatura con la que fueron identificadas las especies. Las formas de vida se determinaron de acuerdo a Steubing et al. (2002). El estado de conservación de las especies se tomó de Benoit (1989) y Hechenleitner et al. (2005). Para las bulbosas se siguió 
a Ravenna et al. (1998) y para los helechos a Baeza et al. (1998). La colección de plantas se encuentra depositada en el herbario del Museo Nacional de Historia Natural (SGO).

Además entre los meses de agosto y octubre del año 2006, se levantaron 30 censos de vegetación de acuerdo a la metodología fitosociológica de la escuela de Zürich-Montpellier (Braun- Blanquet 1979), en parcelas florística, fisonómica y ecológicamente homogéneas de $36 \mathrm{~m}$, tamaño determinado como área mínima para esta comunidad, según la metodología propuestas por Knapp (1984). En cada censo se hizo un inventario exhaustivo de todas las especies presentes (autóctonas e introducidas) y se determinó por simple apreciación visual directa, el porcentaje de cobertura de todos los individuos de cada especie en la parcela, usando la escala de cobertura-abundancia propuesta por Willmanns (1993). Con los 30 censos de vegetación se construyó la tabla fitosociológica inicial, en base a la cual fueron calculadas las frecuencias y coberturas relativas de cada especie (Wikum \& Shanholtzer 1978). La frecuencia relativa se obtuvo sumando las frecuencias absolutas (censos en que están presentes) de todas las especies, llevando el total a 100 y determinando el porcentaje de ese total que le corresponde a cada una. La cobertura relativa se obtuvo sumando las coberturas de todas las especies en los censos en que están presentes, llevando el total a 100 y determinando el porcentaje que le corresponde a cada especie. Finalmente, se sumó la frecuencia relativa y cobertura relativa de cada especie para obtener su valor de importancia. Este valor sirve para jerarquizar las especies de acuerdo a la importancia que tienen dentro de la comunidad analizada, su máximo valor es 200 y no tiene unidad de medida. Tampoco es comparable con valores de importancia obtenidos para las mismas especies en otras comunidades vegetales (Wikum \& Shanholtzer 1978).

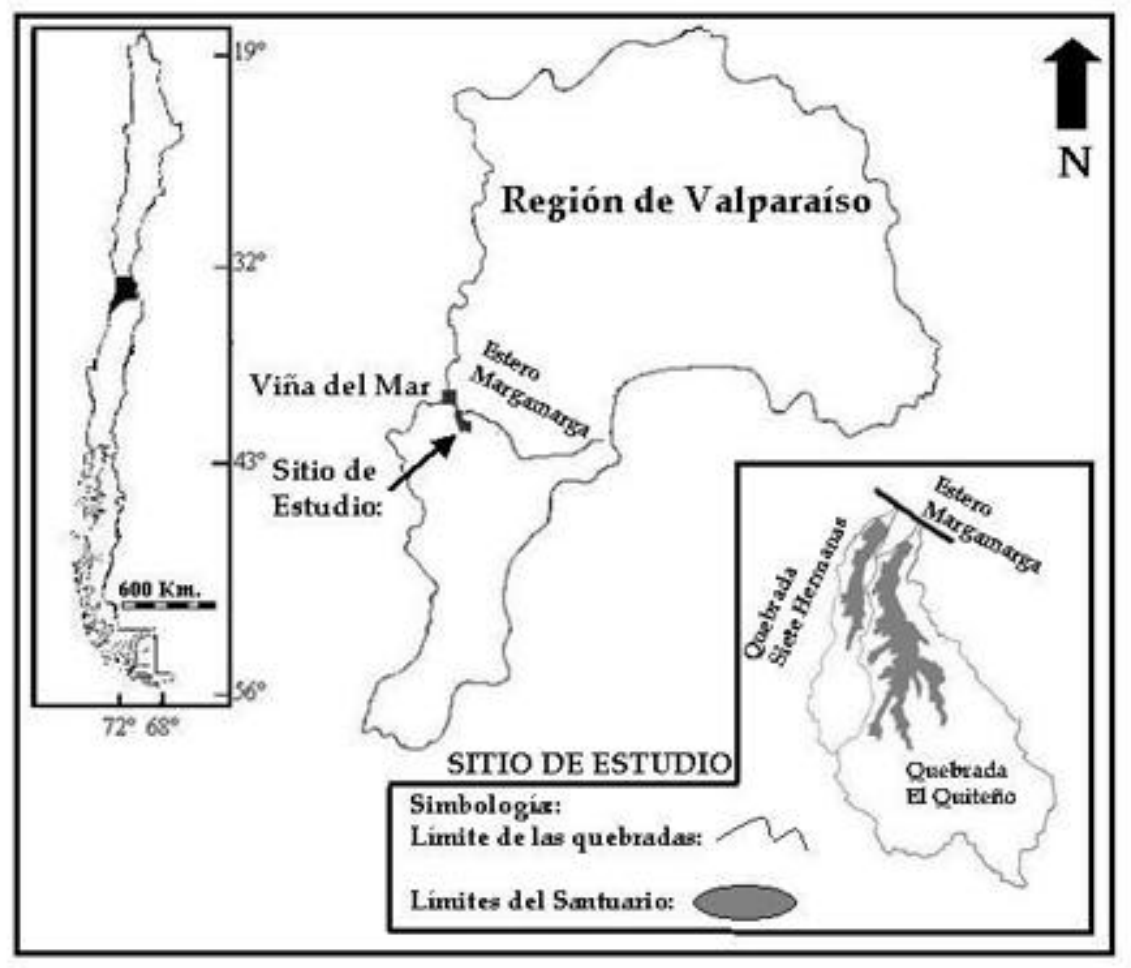

Figura 1. Ubicación del Santuario de la Naturaleza Palmar El Salto, en las quebradas Siete Hermanas y El Quiteño, en la Comuna de Viña del Mar, Región de Valparaíso.

Figure 1. Location of the Nature Sanctuary Palmar El Salto in Viña del Mar, Valparaíso Region. 


\section{RESULTADOS}

RiQueZa DE LA FLORA VASCULAR

De acuerdo a nuestros resultados, la flora vascular del Santuario de la Naturaleza Palmar El Salto se compone de 234 especies (Anexo 1), además de siete elementos hasta ahora identificados solo al nivel de género: Calandrinia, Ranunculus, Schizanthus, Viola, Alstroemeria, Crepis y Juncus.

Las 234 especies se distribuyen en 6 clases, 79 familias y 178 géneros (Tabla I).

Existen tres clases con una sola especie cada una: Sphenopsida con Equisetum bogotense Kunth (yerba del platero), Pinopsida con Pinus radiata D.Don y Gnetopsida con Ephedra chilensis K.Presl (pingo-pingo). La clase mejor representada fue Magnoliopsida con 175 especies que corresponde al 74,79\% de la flora del santuario. Le sigue la clase Liliopsida con 49 especies, lo que representa el 20,94 $\%$ del total. Finalmente la clase Filicopsida presenta 7 especies.

Entre las familias con mayor riqueza de especies se encuentran: Asteraceae con 33 especies, Fabaceae con 15, Poaceae con 14, Apiaceae con 9, Lamiaceae con 8 y finalmente Scrophulariaceae y Orchidaceae con 7 especies cada una.

Tabla I. Distribución taxonómica de las especies del Santuario de la Naturaleza Palmar El Salto.

TABle I. Taxonomical distribution of species the Nature Sanctuary Palmar El Salto.

\begin{tabular}{lllll}
\hline Clase & Familias & Géneros & Especies & \% Especies \\
\hline Sphenopsida & 1 & 1 & 1 & 0,43 \\
Filicopsida & 4 & 5 & 7 & 2,99 \\
Pinopsida & 1 & 1 & 1 & 0,43 \\
Gnetopsida & 1 & 1 & 1 & 0,43 \\
Magnoliopsida & 58 & 138 & 175 & 74,79 \\
Liliopsida & 14 & 32 & 49 & 20,94 \\
\hline
\end{tabular}

\section{FORMAS DE VIDA}

En el catálogo florístico (anexo 1), se incluyen las formas de vida para cada especie, siendo las más abundantes las fanerófitas con 90 especies $(38,46$ $\%)$, seguidas por las hemicriptófitas con 57 especies $(24,363 \%)$. Las terófitas (hierbas anuales) están representadas por 46 especies y las criptófitas por 25 . Los caméfitos son poco relevantes.

\section{ORIGEN FITOGEOGRÁFICO}

De las 234 especies encontradas, el 75,64\% son autóctonas (nativas y endémicas) y sólo el 24,36 \% corresponde a especies introducidas (Figura 2).

El mayor porcentaje de especies introducidas, se encuentra en la Clase Magnoliopsida (Tabla II). Y dentro de esta clase, las familias con el mayor número de especies son Asteraceae, Fabaceae y Lamiaceae con 12,5 y 4 especies respectivamente. La clase Liliopsida reúne sólo 6 especies introducidas, todas de la familia Poaceae.

VALOR DE IMPORTANCIA

La Tabla III muestra las 10 especies más importan- tes del Santuario. De ellas, sólo dos corresponden a especies introducidas, consideradas malezas por Matthei (1995): Euphorbia peplus L. (pichoga) y Fumaria capreolata L., sin embargo, a pesar de que son altamente frecuentes, no poseen gran cobertura. Las 3 especies que tienen más altos valores de importancia corresponden a especies típicas colonizadoras de sectores que han sufrido alteraciones por incendios (Quintanilla \& Reyes 1999; Villaseñor \& Sáiz, 1993). Destaca Nassella chilensis (Trin.) E.Desv. (coironcillo), una herbácea nativa, que no sólo posee el más alto valor de importancia sino que además es la especie más frecuente y de mayor cobertura en el palmar. Jubaea chilensis, la especie que identifica fisonómicamente a la comunidad, es la cuarta de mayor importancia, no tanto por su frecuencia, sino más bien por la cobertura.

ESPECIES EN CATEGORÍA DE CONSERVACIÓN

El Santuario de la Naturaleza Palmar El Salto, alberga nueve especies de plantas amenazadas, la mayoría en categoría de conservación vulnerable (Tabla IV). 
TABLA II. Origen fitogeográfico de las especies ordenado según las clases encontradas en el Santuario de la Naturaleza Palmar El salto.

TABLE II. Phytogeographycal origin of the species, according to the classes found in the Nature Sanctuary Palmar El Salto.

\begin{tabular}{lllll}
\hline & \multicolumn{3}{c}{ Especies } \\
\hline Clase & Autóctonas & $\%$ & Introducidas & $\%$ \\
Sphenopsida & 1 & 100 & 0 & 0 \\
Filicopsida & 7 & 100 & 0 & 0 \\
Pinopsida & 0 & 0 & 1 & 100 \\
Gnetopsida & 1 & 100 & 0 & 0 \\
Magnoliopsida & 125 & 71,43 & 50 & 28,57 \\
Liliopsida & 43 & 87,76 & 6 & 12,24 \\
\hline
\end{tabular}

TABLA III. Especies más importantes del Santuario de la Naturaleza Palmar El Salto.

TABLE III. More important species of the Nature Sanctuary Palmar El Salto.

\begin{tabular}{llll}
\hline Especies & $\begin{array}{l}\text { Frecuencia } \\
\text { relativa }\end{array}$ & $\begin{array}{l}\text { Cobertura } \\
\text { relativa }\end{array}$ & $\begin{array}{l}\text { Valor } \\
\text { de importancia }\end{array}$ \\
\hline Nassella chilensis (Trin.) E.Desv. & 4,62 & 8,68 & 13,31 \\
Chusquea cumingii Nees & 3,47 & 6,78 & 10,25 \\
Retanilla trinervia (Gillies et Hook.) Hook. et Arn. & 3,66 & 5,70 & 9,36 \\
Jubaea chilensis (Molina) Baill. & 2,50 & 6,38 & 8,88 \\
* Euphorbia peplus L. & 4,05 & 2,99 & 7,03 \\
Cryptocarya alba (Molina) Looser & 2,50 & 3,80 & 6,30 \\
Dichondra sericea Sw. & 3,08 & 2,17 & 5,25 \\
Dioscorea parviflora Phil. & 3,08 & 2,17 & 5,25 \\
* Fumaria capreolata L. & 3,08 & 2,17 & 5,25 \\
Podanthus mitiqui Lindl. & 2,12 & 3,12 & 5,24 \\
\hline
\end{tabular}

* Especies introducidas

TABla IV. Especies en alguna categoría de conservación del Santuario de la Naturaleza Palmar El Salto.

TABLE IV. Species in some category of conservation the Nature Sanctuary Palmar El Salto.

\section{Vulnerable}

Nivel nacional: Chloraea cristata Lindl., Chloraea disoides Lindl., Eriosyce curvispina (Bertero ex Colla) Kattermann var. tuberisulcata (Jacobi) Kattermann, Jubaea chilensis (Molina) Baill.

Nivel regional: Blepharocalyx cruckshanksii (Hook. et Arn.) Niedenzu

\section{Rara}

Nivel nacional: Adesmia balsamica Bertero ex Hook. et Arn., Myrceugenia rufa (Colla) Skottsb. ex Kausel Insuficientemente conocida

Nivel nacional: Citronella mucronata (Ruiz et Pav.) D.Don

Nivel regional: Blechnum chilense (Kaulf.) Mett. 


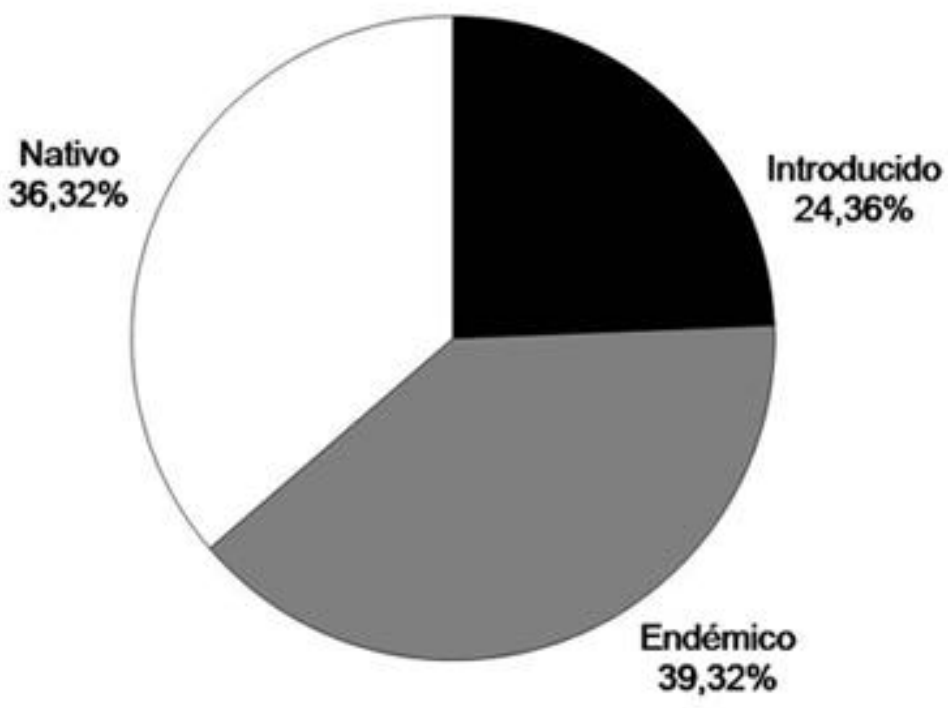

Figura 2. Origen fitogeográfico de la flora del Santuario de la Naturaleza Palmar El Salto.

FIgURE 2. Phytogeographycal origin of the flora from the Nature Sanctuary Palmar El Salto

\section{DISCUSION}

Estudios florísticos realizados por Redón (2003), en zonas cercanas al palmar, revelan la presencia de algo más de cien especies de plantas vasculares creciendo en estos lugares, entre nativas e introducidas. Sin embargo, muy lejos de estas cifras, nuestro estudio revela un total de 241 taxa presentes en el Santuario de la Naturaleza Palmar El Salto, siete determinados a nivel de género y 234 a nivel de especie, lo que representa el $10 \%$ de toda la flora de la región mediterránea de Chile central, la que alberga un total de 2.400 especies de plantas vasculares (Arroyo et al. 1995).

Del total de especies, la familia más numerosa resultó ser Asteraceae, hecho que concuerda plenamente con la situación descrita para Chile continental por Marticorena (1991). En cuanto a la flora introducida, esta se encuentra distribuida en 25 familias, sin embargo Asteraceae y Poaceae conforman el $31,5 \%$ del total de especies consideradas malezas para el Santuario de la Naturaleza Palmar El Salto, porcentaje muy cercano al 35,8\% señalado por Matthei (1995), para estas mismas familias considerando el total de malezas que crecen en el país. Otro punto interesante de destacar es la presencia de Lathyrus latifolius L., especie cultivada en los jardines, que hasta ahora no había sido citada como maleza, pero que aparentemente se escapó de cultivo y se ha establecido exitosamente en algunos sectores de fondo de quebrada en el palmar.

Comparando la flora del Santuario con estudios florísticos en otras áreas protegidas que albergan ecosistemas naturales de la Región de Valparaíso, se evidencia una alta diversidad florística en un área muy reducida. Así, el palmar El Salto, en las 220 ha que abarcó este estudio, cuenta con prácticamente la mitad de las especies que es posible encontrar en el Parque Nacional La Campana, el cual alberga 545 especies en 8.000 ha (Elórtegui \& Moreira 2002).

Muchas de éstas especies son endémicas de Chile mediterráneo: Jubaea chilensis, Phycella ignea (Lindl.) Lindl., Phycella bicolor (Ruiz et Pav.) Herb., Pteromonnina pterocarpa (Ruiz et Pav.) B.Eriksen y las orquídeas Chloraea cristata Lindl. y Chloraea disioides Lindl. Destaca el arbusto Adesmia balsamica, especie endémica de la Región de Valparaíso y hoy en día en peligro de extinción. El lugar alberga además muchos géneros endémicos 
de Chile: Conanthera, Eriosyce, Francoa, Gilliesia, Leucocoryne, Podanthus, Trichopetalum, etc. y varios géneros monotípicos, es decir que tienen sólo una especie en nuestro país, tales como: Chaptalia, Blepharocalyx y Citronella, entre otros. Es destacable el caso de los géneros que además de ser monotípicos, son endémicos de Chile mediterráneo, tales como Peumus, Adenopeltis y Jubaea (Marticorena 1991). Es interesante la presencia de dos especies endémicas de la región mediterránea, cuyo límite norte es la Región de Valparaíso, sin embargo son bastante escasas y difíciles de observar en otros sectores que no sea el Palmar El Salto. Estas son Myrceugenia lanceolata (Juss. ex J. St.-Hil.) Kausel, abundante en los fondos de quebradas, a orillas de los cursos de agua y Adesmia loudonia Hook. et Arn., que habita las partes altas de las cuencas, entre las quebradas Siete Hermanas y El Quiteño. En estos mismos sectores altos se concentran además muchas de las herbáceas endémicas identificadas en este estudio. Sin embargo, cuando se establecieron los límites del santuario, se dejó precisamente estos lugares fuera del área de protección, ya que no se usó un criterio científico, respaldado por un estudio ecológico, sino que solo se hizo un conteo de palmas para definir los sectores con mayor densidad de la especie, criterio considerado suficiente para la delimitación del Santuario de la Naturaleza Palmar El Salto (Möder et al., 1997). Todo lo anterior cobra relevancia si se tiene presente que Chile central es considerado como una de las 34 regiones prioritarias para la conservación de la biodiversidad a escala mundial (Myers et al. 2000; Mittermeier et al.2005).

En los fondos de quebrada, a orillas de los cursos de agua, es interesante destacar la presencia de Chusquea cumingii Nees (quila), con un hábito de crecimiento bastante particular y distinto de las quilas que crecen en las laderas. Los ejemplares del fondo de quebrada son notablemente más altos (hasta $5 \mathrm{~m}$.) y erectos, a diferencia de los ejemplares de las laderas, que no superan los dos metros de altura y crecen más bien tendidos. Además, en la primavera de 2006 y 2007, observamos la floración de individuos aislados de quila de fondo de quebrada, no así la de las laderas. Este tipo de floración aislada para las especies del género Chusquea es común y ya fue mencionado por Matthei (1997), para las especies de la X Región, sin embargo, no deja de llamar la atención que en el palmar sean solo las poblaciones del fondo de quebrada las que florecen. De acuerdo a estas observaciones, sugerimos que debiera hacerse una revisión del género Chusquea para la región mediterránea y determinar si estos dos tipos de quila observados en estas quebradas obedecen sólo a hábitos de crecimiento distintos originados por las condiciones del sitio, o se deben a la presencia de dos taxa en la región.

Las cuatro especies que presentan los más altos valores de importancia en el santuario son aquellas que se caracterizan por colonizar áreas que han sido devastadas por el fuego o que poseen algún tipo de cualidad que les permite resistir a éste tipo de alteraciones (Quintanilla \& Reyes 1999; Villaseñor \& Sáiz, 1993). Así, Nassella chilensis, Chusquea cuminguii y Retanilla trinervia (Gillies et Hook.) Hook. et Arn., son especies autóctonas que actúan como pioneras, pues son las primeras en brotar tras un incendio. Tanto Chusquea cummingii, como Retanilla trinervia forman agrupaciones casi puras en diversos sectores de las laderas de las quebradas, sin distinción de la exposición o de la altura en la que se encuentren, ocupando gran parte del estrato arbustivo del palmar, mientras que Nassella chilensis cubre grandes áreas del estrato herbáceo. Finalmente, Jubaea chilensis ha sobrevivido a este régimen de incendios permanentes porque en estado adulto no se ve afectada por el fuego (Villaseñor \& Sáiz 1993). Por tanto, si bien es cierto que las cuatro especies más importantes del palmar son autóctonas, su frecuencia y cobertura revelan un régimen de incendios que ha alterado las comunidades vegetales originales (Quintanilla \& Reyes 1999). No obstante lo anterior, y debido a la alta diversidad florística del palmar, los endemismos y géneros monotípicos, las especies con problemas de conservación que habitan el lugar, la gran presión antrópica y la existencia de muy pocas áreas protegidas en esta región y menos aun en la comuna de Viña del Mar, es que el Santuario de la Naturaleza Palmar El Salto y sus alrededores representan un verdadero refugio para la sobrevivencia de un gran número de especies vegetales únicas en el mundo.

\section{AGRADECIMIENTOS}

Al proyecto URB -al "Plan maestro Siete Hermanas" de la Ilustre Municipalidad de Viña del Mar por 
financiar parte este trabajo. A Carmen Estay, Alicia Marticorena y Mélica Muñoz por su valiosa ayuda en la elaboración del catálogo florístico. Al Profesor Francisco Sáiz y a dos revisores anónimos por sus observaciones, críticas y sugerencias en la preparación del manuscrito.

\section{BIBLIOGRAFIA}

Arroyo, M., L. Cavieres, C. Marticorena \& M. Muñoz. 1995. Convergence in the Mediterranean Floras in Central Chile and California: Insights from Comparative Biogeography. In: Arroyo MTK, PH Zedler \& MD Fox (Eds.) Ecology and Biogeography of Mediterranean Ecosystems in Chile, California and Australia. Springer Verlag, Ecological Studies 108:43-88.

Baeza, M., E. Barrera, J. Flores, C. Ramírez \& R. RodRíGuez. 1998. Categorías de conservación de Pteridophyta nativas de Chile. Boletín del Museo Nacional de Historia Natural 47:23-46.

BAEZA, V. 1930. Los nombres vulgares de las plantas silvestres de Chile y su concordancia con los nombres científicos. Y observación sobre la aplicación técnica y medicinal de algunas especies. Imprenta El Globo, 272 pp.

Benoit, I. 1989.. Libro Rojo de la Flora Terrestre de Chile, Primera parte. CONAF. Santiago, Chile. 157 pp.

Braun-Blanquet J. 1979. Fitosociología. Bases para el estudio de las comunidades vegetales. H. Blume Ediciones. España. 820 pp.

Donoso, C. 1981. Investigación y Desarrollo Forestal, Tipos forestales de los bosques nativos de Chile. Documento de trabajo $\mathrm{N}^{\circ} 38$. Chile. $82 \mathrm{pp}$.

Elórtegui, S. \& A. Moreira. 2002. Parque Nacional La Campana. Origen de una Reserva de la Biosfera en Chile central. Taller La Era. Santiago. 176 pp.

Gajardo, R. 1994. La Vegetación Natural de Chile. Clasificación y Distribución Geográfica. Editorial Universitaria, Santiago. 165 pp.

Gallardo, H., L. M öder \& I. Benoit. 2006. Plan nacional para la conservación y recuperación de la Palma Chilena. Jubaea chilensis (Mol) Baillon. CONAF. Región de Valparaíso. 20 pp.

González, L., M. T oral \& R. Garfias. 2001. Estudios de poblaciones de Palma chilena: avanzado estado de deterioro. Chile Forestal 284: 53- 56.

Hechenleitner, P., M. G ardner, P. Thomas, C. EcheVerría, B. Escobar, P. Brownless \& C. Martínez. 2005. Plantas Amenazadas del Centro Sur de Chile. Distribución, Conservación y Propagación. Primera Edición. Universidad Austral de Chile y Real Jardín Botánico de Edimburgo. 188 pp.

KNAPP, R. 1984. Considerations on qualitative parameters and qualitative attributes in vegetation analysis and in phytosociological relevés. En: Sampling methods and taxon analysis in vegetation science
(Ed. R.Knapp ), pp. 77-119. Dr. W. Junk Pub., La Haya.

M articorena, C. \& M. Quezada. 1985. Catálogo de la flora vascular de Chile. Gayana Botánica 42: 1157.

M ARticorena, C. 1991. Contribución a la estadística de la flora vascular de Chile. Gayana Botánica 47: 85113.

M Aтthei, O. 1995. Manual de Malezas que crecen en Chile. Alfabeta Impresores, Santiago de Chile. 545 pp.

M Aтthei, O. 1997. Las especies del género Chusquea Kunth (Poaceae: Bambusoideae), que crecen en la X región, Chile. Gayana Botánica 54:199-220.

Michea, G. 1988. Estudio poblacional de Palma chilena en el sector Ocoa, Parque Nacional La Campana. Medio Ambiente 9: 124-130.

Mittermeier, R., P. Robles, M. Hoffman, J. Pilgrim, T. Brooks, C. Mittermeier, J. Lamoreux \& G. da FonseCA . 2005. Hotspots Revisited: Earth's Biologically Richest and Most Endangered Terrestrial Ecoregions. The University of Chicago Press. E.E.U.U. 392 pp.

Möder, L., O. Rojas \& L. Gonzalez. 1997. Reestudio de los límites propuestos para creación del área "Palmar El Salto" como Santuario de la Naturaleza. Informe técnico $N^{\circ} 15 / 97$. U.G. Patrimonio Silvestre CONAF V región. 6 pp.

Myers, N., R. Mittermeier, CG. Mittermeier, G. DA Fonseca \& J. Kent. 2000. Biodiversity hotspots for conservation priorites. Nature 403:853-858

Portal, M. \& M. CaLderón. 1995. Palmar Las 7 HermanasEl Salto. CONAF, Región de Valparaíso, 23 pp.

Quintanilla, V. \& C. Reyes. 1999. Modificaciones por efecto del fuego en el bosque esclerófilo de quebradas húmedas de Chile central y su incidencia en la palma chilena. Revista Geográfica Terra Australis. No 44. I.G.M. Santiago.

Ravenna, P., S. Teillier, J. Macaya, R. Rodríguez \& O. ZöLLnER. 1998. Categorías de conservación de las plantas bulbosas nativas de Chile. Boletín del Museo Nacional de Historia Natural 47:47-68.

Redón, J. 2003. Flora y vegetación de la cuenca del Estero de Viña del Mar, Quinta Región, Chile. Cuadernos de Investigación No2. Universidad de Viña del Mar. $60 \mathrm{pp}$.

Steubing, L., R. Godoy \& M. Alberdi. 2002. Métodos de Ecología Vegetal. Editorial Universitaria. Valdivia, Chile. 345 pp.

Villaseñor, R. \& F. Sáiz. 1993. Incendios forestales en el Parque Nacional La Campana Sector Ocoa, V Región, Chile. Efecto sobre el estrato arbustivoarbóreo. Anales Museo de Historia Natural de Valparaíso, Chile. 21:15-26.

Wikum, D. \& G. Shanholtzer. 1978. Application of the Braun-Blanquet cover-abundance scale for vegetation analysis in land development studies. Environmental Management 2:323-329.

Wilmanns, O. 1993. Ökologische Pflanzensoziologie. $5^{\text {a }}$ edition. Meyer, Heidelberg. 479 pp. 
Anexo I. Catálogo florístico de la flora vascular del Santuario de la Naturaleza Palmar El Salto.

Nombres científicos, Familias, Nombres comunes, Origen fitogeográfico (O.F.), Forma de vida (F.V.) y Estado de conservación (E.C.).

Anexo I. List of the vascular plants of the Nature Sanctuary Palmar El Salto.

Scientific names, Families, Common names, Phytogeographycal origin (O.F.), Life forms (F.V.) and State of conservation (E.C.)

CLASE / Nombre científico

Equisetum bogotense Kunth

\section{FILICOPSIDA}

Adiantum chilense Kaulf.

Adiantum excisum Kunze

Azolla filiculoides Lam.

Blechnum chilense (Kaulf.) Mett.

Blechnum hastatum Kaulf.

Cheilanthes hypoleuca (Kunze) Mett.

Thelypteris argentina (Hieron.) Abbiatti

\section{PINOPSIDA}

Pinus radiata D.Don

\section{GNETOPSIDA}

Ephedra chilensis K.Presl

\section{MAGNOLIOPSIDA}

Acacia dealbata Link.

Acaena pinnatifida Ruiz et Pav.

Acrisione denticulata (Hook. et Arn.) B.Nord.

Adenopeltis serrata (W.T.Aiton) I.M.Johnst.

Adesmia balsámica Bertero ex Hook. et Arn.

Adesmia confusa Ulib.

Adesmia filifolia Clos

Adesmia loudonia Hook. et Arn

Ageratina glechonophylla (Less.) R.M.King et H.Rob.

Alonsoa meridionalis (L.) Kuntze

Amsinckia calycina (Moris) Chater

Anagallis arvensis L.

Apium panul (Bertero ex DC.) Reiche

Aristeguietia salvia (Colla) R.M.King et H.Rob.

Aristolochia chilensis Bridges ex Lidl.

Aristotelia chilensis (Molina) Stuntz

Astragalus amatus Clos

Azara celastrina D.Don

Azorella spinosa (Ruiz et Pav.) Pers.

Baccharis linearis (Ruiz et Pav.) Pers.

Baccharis racemosa (Ruiz et Pav.) DC.

Baccharis salicifolia (Ruiz et Pav.) Pers.

Baccharis vernalis F.H.Hellw.

Berberis actinacantha Mart.

Bidens pilosa $\mathrm{L}$.

Bidens subalternans DC.

Blepharocalyx cruckshanksii (Hook. et Arn.) Niedenzu

Bowlesia uncinata Colla

Calceolaria ascendens Lind.

Calceolaria corymbosa Ruiz et Pav.

Calceolaria thyrsiflora Graham

Calceolaria tripartita Ruiz et Pav.

Cardamine flaccida Cham. et Schltdl.

Familia

Nombre común

O. F. F.V. E.C

Equisetaceae

Adiantaceae

Adiantaceae

Azollaceae

Blechnaceae

Blechnaceae

Adiantaceae

Thelypteridaceae

Pinaceae

Ephedraceae

Mimosaceae

Rosaceae

Asteraceae

Euphorbiaceae

Fabaceae

Fabaceae

Fabaceae

Fabaceae

Asteraceae

Scrophulariaceae

Boraginaceae

Primulaceae

Apiaceae

Asteraceae

Aristolochiaceae

Elaeocarpaceae

Fabaceae

Flacourtiaceae

Apiaceae

Asteraceae

Asteraceae

Asteraceae

Asteraceae

Berberidaceae

Asteraceae

Asteraceae

Myrtaceae

Apiaceae

Scrophulariaceae

Scrophulariaceae

Scrophulariaceae

Scrophulariaceae

Brassicaceae yerba del platero $\mathrm{N} \quad \mathrm{H} \quad-$

culantrillo

costilla de vaca

$\begin{array}{lll}\mathrm{N} & \mathrm{H} & - \\ \mathrm{E} & \mathrm{H} & - \\ \mathrm{N} & \mathrm{T} & - \\ \mathrm{N} & \mathrm{H} & \text { I.C. } \\ \mathrm{N} & \mathrm{H} & \text { F.P. } \\ \mathrm{N} & \mathrm{H} & - \\ \mathrm{N} & \mathrm{H} & -\end{array}$

pingopingo

$\mathrm{N} \quad \mathrm{F} \quad-$

colliguay macho

jarilla

barbón

ajicillo

salvia macho

oreja de zorro

maqui

lilen

hierba santa

romerillo

chilca

michay

palo colorado

capachito

capachito

palito dulce 
Continuación Anexo I.

\begin{tabular}{|c|c|c|c|c|c|}
\hline CLASE / Nombre científico & Familia & Nombre común & O. F. & F.V. & $\overline{\text { E.C }}$ \\
\hline \multicolumn{6}{|l|}{ Cardionema ramosissimum (Weinm.) } \\
\hline A.Nelson et J.F.Mac-br. & Caryophyllaceae & & $\mathrm{N}$ & $\mathrm{H}$ & -- \\
\hline Carduus pycnocephalus L. & Asteraceae & & I & $\mathrm{T}$ & -- \\
\hline Centaurea melitensis L. & Asteraceae & & I & $\mathrm{T}$ & -- \\
\hline Cestrum parqui L'Her. & Solanaceae & palqui & $\mathrm{N}$ & $\mathrm{F}$ & -- \\
\hline Chamaemelum mixtum (L.) All. & Asteraceae & & I & $\mathrm{T}$ & -- \\
\hline \multicolumn{6}{|l|}{ Chaptalia escapa (Pers.) Baker } \\
\hline var. chilensis (DC.) Burkart & Asteraceae & & $\mathrm{E}$ & $\mathrm{H}$ & - \\
\hline Chenopodium ambrosioides L. & Chenopodiaceae & & $\mathrm{N}$ & $\mathrm{F}$ & -- \\
\hline Chrysantemoides molinifera (L.) Norl. & Asteraceae & & I & $\mathrm{F}$ & -- \\
\hline Cichorium intybus L. & Asteraceae & achicoria & I & $\mathrm{T}$ & -- \\
\hline Cissus striata Ruiz et Pav. & Vitaceae & pilpil voqui & $\mathrm{N}$ & $\mathrm{F}$ & -- \\
\hline Citronella mucronata (Ruiz et Pav.) D.Don & Icacinaceae & naranjillo & $\mathrm{E}$ & $\mathrm{F}$ & I.C. \\
\hline Clarkia tenella (Cav.) F.H.Lewis et M.R.Lewis & Onagraceae & huasita & $\mathrm{N}$ & $\mathrm{T}$ & - \\
\hline Colliguaja odorifera Molina & Euphorbiaceae & colliguay & $\mathrm{E}$ & $\mathrm{F}$ & -- \\
\hline Conium maculatum L. & Apiaceae & cicuta & I & $\mathrm{T}$ & -- \\
\hline Conyza floribunda Kunth & Asteraceae & & $\mathrm{N}$ & $\mathrm{T}$ & -- \\
\hline Convolvulus chilensis Pers. & Convolvulaceae & & $\mathrm{E}$ & $\mathrm{F}$ & - \\
\hline Cotula coronopifolia L. & Asteraceae & botón de oro & I & $\mathrm{C}$ & -- \\
\hline Crataegus laevigata (Poir.) DC. & Rosaceae & & I & $\mathrm{F}$ & - \\
\hline Cryptocarya alba (Molina) Looser & Lauraceae & peumo & $\mathrm{E}$ & $\mathrm{F}$ & -- \\
\hline Cuscuta chilensis KerGawl & Cuscutaceae & cabello de ángel & $\mathrm{N}$ & $\mathrm{T}$ & -- \\
\hline Datura ferox $L$. & Solanaceae & & I & $\mathrm{T}$ & _- \\
\hline Dichondra sericea $\mathrm{Sw}$. & Convolvulaceae & & $\mathrm{N}$ & $\mathrm{H}$ & -- \\
\hline Diplolepis menziesii J.H.Schult. & Asclepiadaceae & & $\mathrm{E}$ & $\mathrm{F}$ & -- \\
\hline Drimys winteri J.R. et G. Forster & Winteraceae & canelo & $\mathrm{N}$ & $\mathrm{F}$ & -- \\
\hline Echinopsis chiloensis (Colla) Friedrich et G.D.Rowley & Cactaceae & & $\mathrm{E}$ & $\mathrm{F}$ & - \\
\hline Epilobium ciliatum Raf. & Onagraceae & & $\mathrm{N}$ & $\mathrm{C}$ & -- \\
\hline \multicolumn{6}{|l|}{ Eriosyce curvispina (Bertero ex Colla) Kattermann } \\
\hline var. tuberisulcata (Jacobi) Kattermann & Cactaceae & & $\mathrm{E}$ & $\mathrm{C}$ & $\mathrm{V}$ \\
\hline Erodium botrys (Cav.) Bertol. & Geraniaceae & alfilerillo & I & $\mathrm{T}$ & -- \\
\hline Eryngium paniculatum Cav. et Dombey ex F.Delaroche & Apiaceae & cardoncillo & $\mathrm{N}$ & $\mathrm{H}$ & - \\
\hline Escallonia illinita K.Presl. & Escalloniaceae & barraco & $\mathrm{E}$ & $\mathrm{F}$ & -- \\
\hline Escallonia pulverulenta (Ruiz et Pav.) Pers. & Escalloniaceae & corontillo & $\mathrm{E}$ & $\mathrm{F}$ & -- \\
\hline Escallonia rubra (Ruiz et Pav.) Pers. & Escalloniaceae & & $\mathrm{N}$ & $\mathrm{F}$ & -- \\
\hline Eschscholzia californica Cham. & Papaveraceae & dedal de oro & I & $\mathrm{H}$ & -- \\
\hline Eucalyptus globulus Labill. & Myrtaceae & & I & $\mathrm{F}$ & - \\
\hline Euphorbia lathyris L. & Euphorbiaceae & matarratones & I & $\mathrm{F}$ & -- \\
\hline Euphorbia peplus L. & Euphorbiaceae & pichoa & I & $\mathrm{T}$ & - \\
\hline Foeniculum vulgare Mill. & Apiaceae & & I & $\mathrm{F}$ & -- \\
\hline Francoa appendiculata Cav. & Francoaceae & llaupanke & $\mathrm{E}$ & $\mathrm{Cr}$ & -- \\
\hline Fuchsia magellanica Lam. & Onagraceae & & $\mathrm{N}$ & $\mathrm{F}$ & -- \\
\hline Fumaria capreolata L. & Fumariaceae & & I & $\mathrm{T}$ & - \\
\hline Galega officinalis L. & Fabaceae & & I & $\mathrm{F}$ & -- \\
\hline Galium aparine L. & Rubiaceae & lengua de gato & I & $\mathrm{T}$ & -- \\
\hline Galium hypocarpium (L.) Endl. ex Griseb. & Rubiaceae & & $\mathrm{N}$ & $\mathrm{F}$ & -- \\
\hline Galium suffruticosum Hook. et Arn. & Rubiaceae & & $\mathrm{E}$ & $\mathrm{F}$ & - \\
\hline Gamochaeta coarctata (Willd.) Kerguélen & Asteraceae & & $\mathrm{N}$ & $\mathrm{T}$ & -- \\
\hline Gamochaeta stachydifolia (Lam.) Cabrera & Asteraceae & & $\mathrm{N}$ & $\mathrm{C}$ & -- \\
\hline Geranium berteroanum Colla & Geraniaceae & & $\mathrm{N}$ & $\mathrm{H}$ & -- \\
\hline Geranium core-core Steud. & Geraniaceae & & $\mathrm{N}$ & $\mathrm{H}$ & - \\
\hline Geranium robertianum L. & Geraniaceae & core-core & I & $\mathrm{T}$ & -- \\
\hline Glandularia laciniata (L.) Schnack et Covas & Verbenaceae & & $\mathrm{N}$ & $\mathrm{H}$ & -- \\
\hline Gnaphalium philippi Cabrera & Asteraceae & & $\mathrm{N}$ & $\mathrm{C}$ & - \\
\hline Gnaphalium viravira Molina & Asteraceae & hierba de la vida & $\mathrm{E}$ & $\mathrm{C}$ & -- \\
\hline Gochnatia foliolosa (D.Don) D.Don ex Hook. et Arn. & Asteraceae & & $\mathrm{E}$ & $\mathrm{F}$ & -- \\
\hline Gunnera tinctoria (Molina) Mirb. & Gunneraceae & & $\mathrm{N}$ & $\mathrm{H}$ & -- \\
\hline Hirschfeldia incana (L.) Lagr.-Foss. & Brassicaceae & & I & $\mathrm{T}$ & -- \\
\hline Hydrocotyle modesta Cham. et Schltdl. & Apiaceae & & $\mathrm{N}$ & $\mathrm{H}$ & -- \\
\hline
\end{tabular}


Continuación Anexo I.

\begin{tabular}{|c|c|c|c|c|c|}
\hline CLASE / Nombre científico & Familia & Nombre común & O. F. & F.V. & E.C. \\
\hline Hypochaeris radicata $\mathrm{L}$. & Asteraceae & hierba del chancho & I & $\mathrm{H}$ & -- \\
\hline Hypochaeris scorzonerae (DC.) F.Muell & Asteraceae & & E & $\mathrm{H}$ & -- \\
\hline Kageneckia oblonga Ruiz et Pav. & Rosaceae & bollén & $\mathrm{E}$ & $\mathrm{F}$ & -- \\
\hline Lamiun amplexicaule L. & Lamiaceae & & I & $\mathrm{T}$ & - \\
\hline Lardizabala biternata Ruiz et Pav. & Lardizabalaceae & coile & E & $\mathrm{F}$ & -- \\
\hline Lathyrus berteroanus Colla & Fabaceae & clarincillo & E & $\mathrm{F}$ & -- \\
\hline Lathyrus latifolius L. & Fabaceae & & I & $\mathrm{F}$ & -- \\
\hline Lepidium strictum (S.Watson) Rattan & Brassicaceae & & $\mathrm{N}$ & $\mathrm{T}$ & -- \\
\hline Lithrea caustica (Molina) Hook. et Arn. & Anacardiaceae & litre & $\mathrm{E}$ & $\mathrm{F}$ & -- \\
\hline Loasa triloba Dombey ex Juss. & Loasaceae & ortiga brava & $\mathrm{E}$ & $\mathrm{T}$ & - \\
\hline Lobelia excelsa Bonpl. & Lobeliaceae & & E & $\mathrm{F}$ & -- \\
\hline Ludwigia peploides (Kunth) P.H.Raven subsp. & & & & & \\
\hline montevidensis (Spreng.) P.H.Raven & Onagraceae & & E & $\mathrm{Cr}$ & - \\
\hline Lupinus arboreus Sims & Fabaceae & chocho & I & $\mathrm{F}$ & -- \\
\hline Margyricarpus pinnatus (Lam.) Kuntze & Rosaceae & & $\mathrm{N}$ & $\mathrm{H}$ & -- \\
\hline Marrubium vulgare L. & Lamiaceae & toronjil cuyano & I & $\mathrm{C}$ & - \\
\hline Maytenus boaria Molina & Celastraceae & maiten & $\mathrm{N}$ & $\mathrm{F}$ & - \\
\hline Menonvillea linearis DC. & Brassicaceae & & $\mathrm{E}$ & $\mathrm{T}$ & - \\
\hline Mentha piperita L. & Lamiaceae & hierba buena & I & $\mathrm{C}$ & -- \\
\hline Mentha pulegium L. & Lamiaceae & poleo & I & $\mathrm{C}$ & -- \\
\hline Mimulus glabratus Kunth. & Scrophulariaceae & & $\mathrm{N}$ & $\mathrm{C}$ & - \\
\hline Modiola caroliniana (L.) G.Don & Malvaceae & & I & $\mathrm{H}$ & - \\
\hline Muehlenbeckia hastulata (Sm.) I.M.Johnst. & Polygonaceae & quilo & $\mathrm{N}$ & $\mathrm{F}$ & -- \\
\hline Mutisia subulata Ruiz et Pav. & Asteraceae & clavel del campo & $\mathrm{N}$ & $\mathrm{F}$ & -- \\
\hline Myoschilos oblonga Ruiz et Pav. & Santalaceae & & $\mathrm{N}$ & $\mathrm{F}$ & -- \\
\hline Myrceugenia exsucca (DC.) O.Berg & Myrtaceae & temu & $\mathrm{N}$ & $\mathrm{F}$ & -- \\
\hline Myrceugenia lanceolata (Juss. ex J. St.-Hil.) Kausel & Myrtaceae & & $\mathrm{E}$ & $\mathrm{F}$ & -- \\
\hline Myrceugenia obtusa (DC.) O.Berg & Myrtaceae & arrayán & E & $\mathrm{F}$ & -- \\
\hline Myrceugenia rufa (Colla) Skottsb. ex Kausel & Myrtaceae & & $\mathrm{E}$ & $\mathrm{F}$ & P.E. \\
\hline Oenothera stricta Ledeb. ex Link & Onagraceae & & $\mathrm{N}$ & $\mathrm{T}$ & -- \\
\hline Otholobium glandulosum (L.) J.W.Grimes & Fabaceae & culén & $\mathrm{N}$ & $\mathrm{F}$ & - \\
\hline Oxalis laxa Hook. et Arn. & Oxalidaceae & vinagrillo & $\mathrm{N}$ & $\mathrm{T}$ & - \\
\hline Oxalis rosea Jacq. & Oxalidaceae & culle colorado & $\mathrm{E}$ & $\mathrm{T}$ & -- \\
\hline Parietaria judaica L. & Urticaceae & & I & $\mathrm{C}$ & - \\
\hline Peumus boldus Molina & Monimiaceae & boldo & $\mathrm{E}$ & $\mathrm{F}$ & - \\
\hline Phacelia secunda J.F.Gmel. & Hydrophyllaceae & & $\mathrm{N}$ & $\mathrm{H}$ & -- \\
\hline Pittosporum tobira Ait. & Pittosporaceae & & I & $\mathrm{F}$ & - \\
\hline Pittosporum undulatum Vent. & Pittosporaceae & & I & $\mathrm{F}$ & - \\
\hline Plantago lanceolata L. & Plantaginaceae & siete venas & I & $\mathrm{H}$ & - \\
\hline Podanthus mitiqui Lindl. & Asteraceae & mitique & E & $\mathrm{F}$ & - \\
\hline Polycarpon tetraphyllum (L.) L. & Caryophyllaceae & & I & $\mathrm{T}$ & -- \\
\hline Polygala gayi A.W.Benn. & Polygalaceae & & E & $\mathrm{T}$ & - \\
\hline Polygala gnidioides Willd. & Polygalaceae & & $\mathrm{E}$ & $\mathrm{F}$ & -- \\
\hline Polygonum persicaria L. & Polygonaceae & & I & $\mathrm{T}$ & - \\
\hline Proustia pyrifolia DC. & Asteraceae & parrilla blanca & $\mathrm{E}$ & $\mathrm{F}$ & - \\
\hline Pteromonnina pterocarpa (Ruiz et Pav.) B.Eriksen & Polygalaceae & & $\mathrm{N}$ & $\mathrm{C}$ & - \\
\hline Quillaja saponaria Molina & Rosaceae & quillay & $\mathrm{E}$ & $\mathrm{F}$ & - \\
\hline Quinchamalium chilense Molina & Santalaceae & quinchamalí & $\mathrm{N}$ & $\mathrm{H}$ & - \\
\hline Retanilla trinervia (Gillies et Hook.) Hook. et Arn. & Rhamnaceae & trebo & $\mathrm{E}$ & $\mathrm{F}$ & - \\
\hline Ribes punctatum Ruiz et Pav. & Saxifragaceae & zarzaparrilla & $\mathrm{N}$ & $\mathrm{F}$ & - \\
\hline Rubus ulmifolius Schott. & Rosaceae & zarzamora & I & $\mathrm{F}$ & - \\
\hline Rumex acetosella L. & Polygonaceae & vinagrillo & I & $\mathrm{H}$ & - \\
\hline Salix babylonica L. & Salicaceae & & I & $\mathrm{F}$ & - \\
\hline Salix caprea L. & Salicaceae & & I & $\mathrm{F}$ & - \\
\hline Salix humboldtiana Willd. & Salicaceae & sauce amargo & $\mathrm{N}$ & $\mathrm{F}$ & - \\
\hline Sanicula crassicaulis Poepp. ex DC. & Apiaceae & pata de león & $\mathrm{N}$ & $\mathrm{H}$ & - \\
\hline Sanicula graveolens Poepp. ex DC. & Apiaceae & & $\mathrm{N}$ & $\mathrm{H}$ & - \\
\hline Satureja gilliesii (Graham) Briq. & Lamiaceae & oreganillo & $\mathrm{E}$ & $\mathrm{F}$ & -- \\
\hline Schinus latifolius (Gillies ex Lindl.) Engler & Anacardiaceae & molle & E & $\mathrm{F}$ & - \\
\hline
\end{tabular}


Continuación Anexo I.

\begin{tabular}{|c|c|c|c|c|c|}
\hline CLASE / Nombre científico & Familia & Nombre común & O. F. & F.V. & E.C. \\
\hline $\begin{array}{l}\text { Schinus montanus (Phil.) Engler } \\
\end{array}$ & Anacardiaceae & & E & $\mathrm{F}$ & - \\
\hline Senecio arnicoides Hook. et Arn. & Asteraceae & & $\mathrm{E}$ & $\mathrm{H}$ & -- \\
\hline Senecio glabratus Hook. et Arn. & Asteraceae & & E & $\mathrm{H}$ & -- \\
\hline Senna huidobriana (Phil.) Zoellner et San Martin & Caesalpiniaceae & & E & $\mathrm{F}$ & -- \\
\hline Silene gallica L. & Caryophyllaceae & calabacillo & I & $\mathrm{T}$ & -- \\
\hline Silybum marianum (L.) Gaerth. & Asteraceae & & I & $\mathrm{H}$ & -- \\
\hline Solanum bridgesii Phil. & Solanaceae & & $\mathrm{N}$ & $\mathrm{C}$ & -- \\
\hline Solanum maglia Schltdl. & Solanaceae & & $\mathrm{N}$ & $\mathrm{Cr}$ & -- \\
\hline Sonchus asper (L.) .Hill & Asteraceae & ñilhue & I & $\mathrm{H}$ & -- \\
\hline Sonchus oleraceus L. & Asteraceae & & I & $\mathrm{H}$ & -- \\
\hline Sophora macrocarpa Sm. & Fabaceae & mayú & E & $\mathrm{F}$ & -- \\
\hline Spartium junceum L. & Fabaceae & retamo & I & $\mathrm{F}$ & -- \\
\hline Spergularia villosa (Pers.) Cambess & Caryophyllaceae & & $\mathrm{N}$ & $\mathrm{C}$ & -- \\
\hline Sphacele salviae (Lindl.) Briq. & Lamiaceae & & $\mathrm{E}$ & $\mathrm{F}$ & -- \\
\hline Stachys grandidentata Lindl. & Lamiaceae & hierba santa & $\mathrm{E}$ & $\mathrm{H}$ & -- \\
\hline Stellaria chilensis Pedersen & Caryophyllaceae & & $\mathrm{N}$ & $\mathrm{T}$ & - \\
\hline $\begin{array}{l}\text { Stemodia durantifolia (L.) Sw. var. } \\
\text { chilensis (Benth.) C.C.Cowan }\end{array}$ & Scrophulariaceae & & $\mathrm{N}$ & $\mathrm{F}$ & -- \\
\hline Stenandrium dulce (Cav.) Nees & Acanthaceae & & $\mathrm{N}$ & $\mathrm{Cr}$ & - \\
\hline Teline monspessulana (L.) K.Koch & Fabaceae & & I & $\mathrm{F}$ & - \\
\hline Teucrium bicolor $\mathrm{Sm}$. & Lamiaceae & & $\mathrm{E}$ & $\mathrm{F}$ & - \\
\hline Triptilion spinosum Ruiz et Pav. & Asteraceae & siempreviva & $\mathrm{E}$ & $\mathrm{Cr}$ & - \\
\hline Tropaeolum majus L. & Tropaeolaceae & espuela de galán & I & $\mathrm{F}$ & -- \\
\hline Tropaeolum tricolor Sweet. & Tropaeolaceae & soldadillo & $\mathrm{E}$ & $\mathrm{F}$ & - \\
\hline Valeriana bridgesii Hook. et Arn. & Valerianaceae & & $\mathrm{E}$ & $\mathrm{H}$ & -- \\
\hline Valeriana vaga Clos & Valerianaceae & & E & $\mathrm{H}$ & - \\
\hline Verbena litoralis Kunth & Verbenaceae & & $\mathrm{N}$ & $\mathrm{F}$ & - \\
\hline Vicia nigricans Hook. et Arn. & Fabaceae & & $\mathrm{N}$ & $\mathrm{T}$ & - \\
\hline Vicia vicina Clos & Fabaceae & & $\mathrm{E}$ & $\mathrm{T}$ & -- \\
\hline \multicolumn{6}{|l|}{ LILIOPSIDA } \\
\hline Alstroemeria ligtu L. subsp. simsii (Spreng.) Ehr.Bayer & Alstroemeriaceae & & $\mathrm{E}$ & $\mathrm{Cr}$ & F.P. \\
\hline Avena barbata Pott ex Link & Poaceae & teatina & I & $\mathrm{T}$ & - \\
\hline Briza maxima L. & Poaceae & & I & $\mathrm{T}$ & -- \\
\hline Briza minor L. & Poaceae & tembladera & I & $\mathrm{T}$ & - \\
\hline Bromus berterianus Colla & Poaceae & pasto largo & $\mathrm{N}$ & $\mathrm{T}$ & -- \\
\hline Bromus catharticus Vahl & Poaceae & & $\mathrm{N}$ & $\mathrm{T}$ & -- \\
\hline Bromus hordeaceus L. & Poaceae & & I & $\mathrm{T}$ & - \\
\hline $\begin{array}{l}\text { Carex pseudocyperus L. subsp. haenkeana } \\
\text { (J.Presl et K.Presl) Kük. }\end{array}$ & Cyperaceae & & $\mathrm{N}$ & $\mathrm{H}$ & - \\
\hline Carex riparia Curtis var. chilensis (Brong.) Kük. & Cyperaceae & & $\mathrm{N}$ & $\mathrm{H}$ & - \\
\hline Carex setifolia Kunze & Cyperaceae & & $\mathrm{N}$ & $\mathrm{H}$ & - \\
\hline Chascolytrum subaristatum (Lam.) Desv. & Poaceae & & $\mathrm{N}$ & $\mathrm{H}$ & -- \\
\hline Chloraea bletioides Lindl. & Orchidaceae & & $\mathrm{E}$ & $\mathrm{Cr}$ & _- \\
\hline Chloraea cristata Lindl. & Orchidaceae & & $\mathrm{E}$ & $\mathrm{Cr}$ & $\mathrm{V}$ \\
\hline Chloraea disoides Lindl. & Orchidaceae & & $\mathrm{E}$ & $\mathrm{Cr}$ & $\mathrm{V}$ \\
\hline Chloraea galeata Lindl. & Orchidaceae & & E & $\mathrm{Cr}$ & - \\
\hline Chloraea gavilu Lindl. & Orchidaceae & & $\mathrm{E}$ & $\mathrm{Cr}$ & -- \\
\hline Chloraea multiflora Lindl. & Orchidaceae & & $\mathrm{E}$ & $\mathrm{Cr}$ & - \\
\hline Chusquea cumingii Nees & Poaceae & quila & E & $\mathrm{F}$ & -- \\
\hline Conanthera bifolia Ruiz et Pav. & Tecophilaeaceae & pajarito del campo & $\mathrm{E}$ & $\mathrm{Cr}$ & F.P. \\
\hline Cyperus eragrostis Lam. & Cyperaceae & & $\mathrm{N}$ & $\mathrm{H}$ & - \\
\hline Dioscorea aristolochiaefolia Poepp. & Dioscoraceae & & $\mathrm{E}$ & $\mathrm{F}$ & - \\
\hline Dioscorea bryoniaefolia Poepp. & Dioscoraceae & camisilla & $\mathrm{E}$ & $\mathrm{F}$ & -- \\
\hline Dioscorea parviflora Phil. & Dioscoraceae & & $\mathrm{E}$ & $\mathrm{F}$ & - \\
\hline Dioscorea saxatilis Poepp. & Dioscoraceae & & E & $\mathrm{F}$ & -- \\
\hline Gavilea longibracteata (Lindl.) Sparre ex L.E.Navas & Orchidaceae & & E & $\mathrm{Cr}$ & -- \\
\hline Gilliesia graminea Lindl. & Alliaceae & & $\mathrm{E}$ & $\mathrm{Cr}$ & -- \\
\hline Jubaea chilensis (Molina) Baill. & Arecaceae & palma chilena & $\mathrm{E}$ & $\mathrm{F}$ & $\mathrm{V}$ \\
\hline Juncus bufonius L. & Juncaceae & & $\mathrm{N}$ & $\mathrm{T}$ & - \\
\hline
\end{tabular}


Continuación Anexo I.

\begin{tabular}{|c|c|c|c|c|c|}
\hline CLASE / Nombre científico & Familia & Nombre común & O. F. & F.V. & E.C. \\
\hline Juncus capillaceous Lam. & Juncaceae & & $\mathrm{N}$ & $\mathrm{H}$ & $\overline{-}$ \\
\hline Juncus pallescens Lam. & Juncaceae & & $\mathrm{N}$ & $\mathrm{H}$ & - \\
\hline Leucocoryne violacescens Phil. & Alliaceae & huille & E & $\mathrm{Cr}$ & -- \\
\hline Nassella chilensis (Trin.) E.Desv. & Poaceae & coironcillo & $\mathrm{N}$ & $\mathrm{H}$ & - \\
\hline Olsynium junceum (E.Mey. ex K.Presl.) Goldblatt & Iridaceae & & $\mathrm{N}$ & $\mathrm{Cr}$ & - \\
\hline Pasithea caerulea (Ruiz et Pav.) D.Don & Hemerocallidaceae & azulillo & $\mathrm{N}$ & $\mathrm{Cr}$ & F.P. \\
\hline Paspalum dasypleurum Kunze ex. E.Desv. & Poaceae & & $\mathrm{N}$ & $\mathrm{H}$ & -- \\
\hline Phycella bicolor (Ruiz et Pav.) Herb. & Amaryllidaceae & & $\mathrm{E}$ & $\mathrm{Cr}$ & - \\
\hline Phycella ignea (Lindl.) Lindl. & Amaryllidaceae & & E & $\mathrm{Cr}$ & - \\
\hline Piptatherum miliacea (L.) Coss. & Poaceae & & I & $\mathrm{H}$ & - \\
\hline Poa bonariensis (Lam.) Kunth & Poaceae & & $\mathrm{N}$ & $\mathrm{H}$ & - \\
\hline Polypogon australis Brongn. & Poaceae & & $\mathrm{N}$ & $\mathrm{H}$ & - \\
\hline Puya berteroniana Mez. & Bromeliaceae & chagual & $\mathrm{E}$ & $\mathrm{H}$ & - \\
\hline Puya chilensis Molina & Bromeliaceae & puya & E & $\mathrm{H}$ & - \\
\hline Scirpus asper J.Presl et K. Presl. & Cyperaceae & & $\mathrm{N}$ & $\mathrm{H}$ & - \\
\hline Setaria parviflora (Poir.) Kerquélen & Poaceae & & I & $\mathrm{Cr}$ & - \\
\hline Sisyrinchium striatum Sm. & Iridaceae & & E & $\mathrm{Cr}$ & - \\
\hline Solenomelus pedunculatus (Gillies ex Hook.) Hochr. & Iridaceae & & $\mathrm{E}$ & $\mathrm{Cr}$ & - \\
\hline Trichopetalum plumosum (Ruiz et Pav.) J.F.Macbr. & Laxmanniaceae & & E & $\mathrm{Cr}$ & F.P. \\
\hline Tristagma bibalve (Lindl.) Traub. & Alliaceae & & E & $\mathrm{Cr}$ & F.P. \\
\hline Uncinia phleoides (Cav.) Pers & Cyperaceae & & $\mathrm{N}$ & $\mathrm{H}$ & -- \\
\hline
\end{tabular}

Origen fitogeográfico: Endémico (E), Nativo (N), Introducido (I)

Forma de vida: Fanerófito (F), Caméfito (C), Hemicriptófito (H), Criptófito (Cr), Terófito (T)

Estado de conservación: Vulnerable (V), Peligro de extinción (P.E.), Insuficientemente conocida (I.C.), Fuera de peligro

(F.P.), No evaluado (一).

Recibido:

Aceptado: 